\title{
Mitral valve endocarditis caused by Staphylococcus aureus
}

\author{
Ashok Kumar Pannu MD, S. Prethiviraj MBBS, Archana Angrup MD
}

Cite as: CMAJ 2019 October 15;191:E1137. doi: 10.1503/cmaj.190641

A 35-year-old man who was an intravenous drug user was admitted to hospital (with high-grade fever (about $40^{\circ} \mathrm{C}$ ) and rapidly progressive dyspnea for 1 week. He had peripheral embolic phenomena: Janeway lesions (multiple nontender maculopapular rashes on the tips of the fingers; Figure 1B), subungual hemorrhages (Figure 1C) and conjunctival hemorrhage (Figure 1D). Cardiac auscultation showed a high-pitched, apical systolic murmur of grade 3 . On transthoracic echocardiography, we found a mobile vegetation $\left(20 \times 16 \mathrm{~mm}^{2}\right.$ in size) on the anterior leaflet of the mitral valve (Figure $1 \mathrm{~A}$; a video is available in Appendix 1 , at www. cmaj.ca/lookup/suppl/doi:10.1503/cmaj.190641/-/ DC1). The patient was started empirically on vancomycin and gentamycin administered intravenously. Three blood samples taken for culture from different venipuncture sites grew methicillin-resistant Staphylococcus aureus. His condition rapidly deteriorated and he died of refractory septic shock.

Several factors increase the likelihood of septic embolization in endocarditis: valve affected, infecting organism, and size and mobility of the vegetation. Endocarditis caused by S. aureus and involving the mitral valve (especially the anterior leaf), with a larger (> $10 \mathrm{~mm}$ ) mobile vegetation independently carries a high risk of septic embolization, as seen in our patient. ${ }^{1,2}$
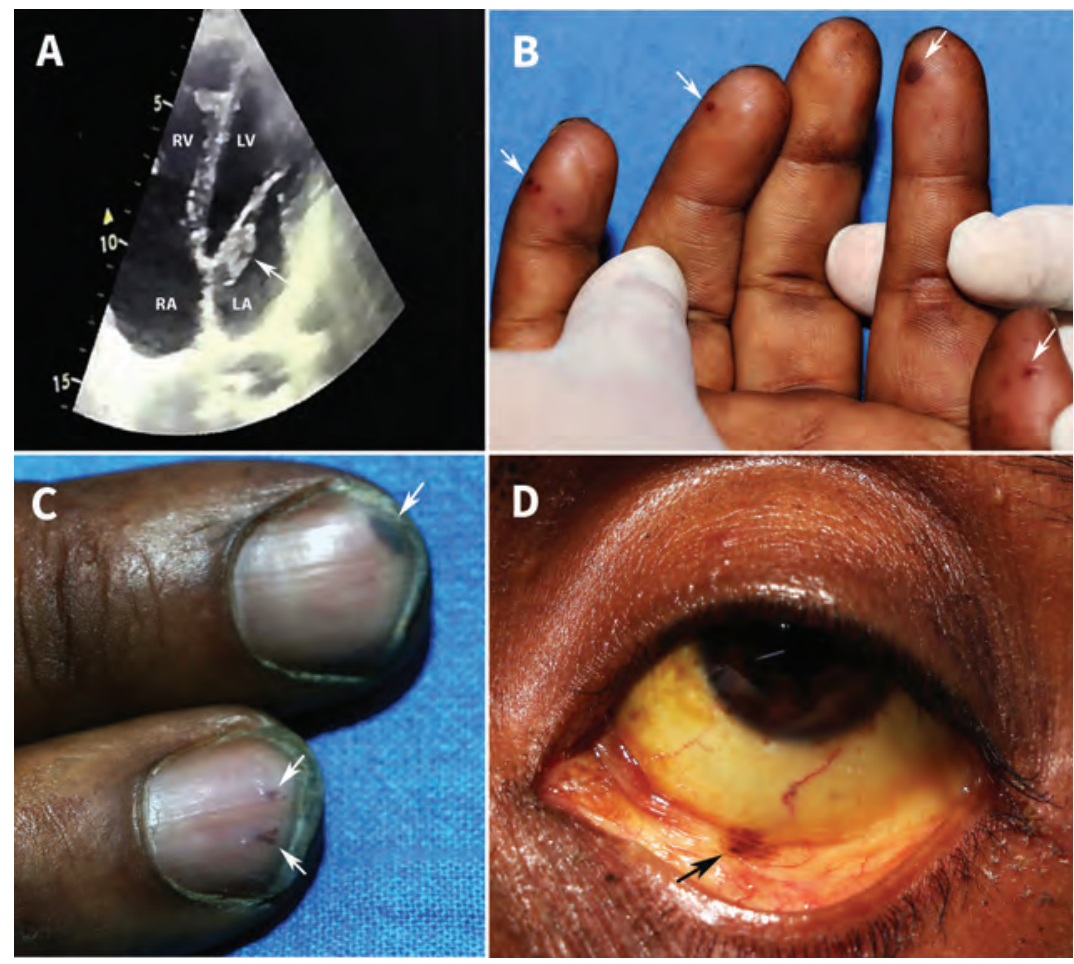

Figure 1: Images from a 35-year-old man with mitral valve endocarditis caused by Staphylococcus aureus: A) apical 4-chamber view on transthoracic echocardiography showing large vegetation ( $20 \times 16 \mathrm{~mm}^{2}$ in size) on the anterior leaflet of the mitral valve, B) Janeway lesions, C) subungual hemorrhages and D) conjunctival hemorrhage.

\section{Reference}

1. Baddour LM, Wilson WR, Bayer AS, et al. Infective endocarditis in adults: Diagnosis, antimicrobial therapy, and management of complications: a scientific statement for healthcare professionals from the American Heart Association. Circulation 2015;132:1435-86.

2. Kasper D, Fauci A, Hauser S, editors. Harrison's Principles of Internal Medicine. 19th ed. New York: McGraw-Hill Education; 2015:924.

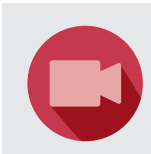

A video of transthoracic echocardiography in a 35-year-old man with endocarditis is available in Appendix 1 , at www.cmaj.ca/lookup/suppl/doi:10.1503/cmaj.190641/-/DC1

\section{Competing interests: None declared.}

This article has been peer reviewed.

The authors have obtained patient consent.
Affiliations: Departments of Internal Medicine (Pannu, Prethiviraj) and Medical Microbiology (Angrup), Post Graduate Institute of Medical Education and Research, Chandigarh, India
Correspondence to: Ashok Kumar Pannu, gawaribacchi@gmail.com 